livraisons

d'Histoire

de l'Architecture

\section{Livraisons de l'histoire de l'architecture}

$17 \mid 2009$

Émotions patrimoniales I

\title{
Réinventer son patrimoine : du vent et des larmes pour le parc du château de Versailles
}

Reinventing Heritage: Wind and Tears in the Park of the Chateau of Versailles

Kulturerbe neu erfinden : Wind und Tränen um den Park des Schlosses von

Versailles

\section{Véronique Dassié}

\section{CpenEdition}

Journals

Édition électronique

URL : http://journals.openedition.org/lha/202

DOI : $10.4000 /$ /ha.202

ISSN : 1960-5994

Éditeur

Association Livraisons d'histoire de l'architecture - LHA

Édition imprimée

Date de publication : 10 juin 2009

Pagination : $27-40$

ISSN : 1627-4970

Référence électronique

Véronique Dassié, «Réinventer son patrimoine : du vent et des larmes pour le parc du château de Versailles », Livraisons de l'histoire de l'architecture [En ligne], 17 | 2009, mis en ligne le 10 juin 2011, consulté le 21 mars 2020. URL : http://journals.openedition.org/lha/202 ; DOI : https://doi.org/ 10.4000//ha.202

Ce document a été généré automatiquement le 21 mars 2020

Tous droits réservés à l'Association LHA 


\section{Réinventer son patrimoine : du vent et des larmes pour le parc du château de Versailles}

Reinventing Heritage: Wind and Tears in the Park of the Chateau of Versailles Kulturerbe neu erfinden: Wind und Tränen um den Park des Schlosses von Versailles

\section{Véronique Dassié} En décembre 1999, une tempête balayait la France et mettait à terre 10000 arbres dans
le parc du château de Versailles ${ }^{1}$ (ill. 1). 


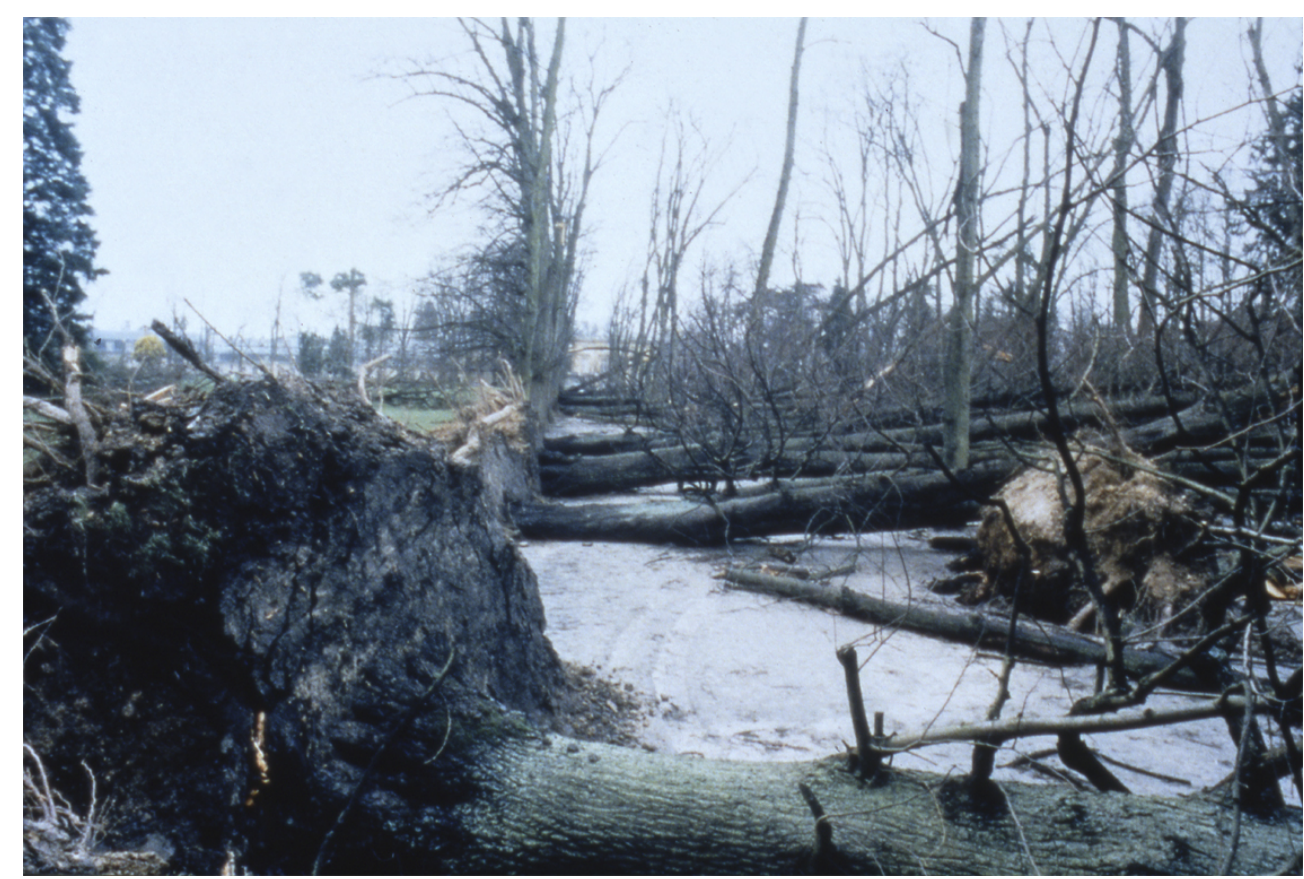

Photographies du parc sur le site Internet du château de Versailles, http://

www.chateauversailles.fr:tempete/Fr/souscription.asp lors de l'appel à souscription internationale, 1999-2001

Cl. J.-M. Manaï.

2 Malgré des dégâts considérables sur les forêts, les infrastructures ferroviaires, électriques et téléphoniques dans toute la France, la destruction de ce lieu emblématique du patrimoine a largement occupé le devant de la scène médiatique. Dès le lendemain de la tempête, les reportages se sont multipliés, faisant du parc détruit le «symbole du désastre $»^{2}$. Pour autant, les journalistes n'ont pas été les seuls à s'émouvoir à son sujet. Au château, des messages de solidarité du monde entier ont rapidement afflué via l'Internet et, tout au long de l'année 2000, des anonymes ont proposé d'aider à la replantation du site.

3 Dire d'un monument historique tel que le domaine de Versailles qu'il relève du patrimoine semble aller de soi, au point que les critères permettant d'établir son " état " patrimonial n'aient pas besoin d'être explicités. Pourtant, la manière dont les individus ordinaires vivent le patrimoine, lui donnent sens et s'attachent à un site en particulier reste dans une large mesure une énigme. L'existence d'un bien collectif prend corps à travers l'idée même de conservation, impérative et évidente, pensée pour les générations futures. Ce devoir de conservation se concrétise parfois à travers une véritable mobilisation, une "émotion patrimoniale " ${ }^{3}$, comme ce fut le cas à propos des arbres du parc à Versailles. L'émotion qui prend forme permet alors de saisir les pôles d'ancrage du lien patrimonial, non seulement du point de vue des institutions qui en sont les garantes, mais aussi pour ses dépositaires anonymes, un collectif devenu acteur de sa reconstruction. Ce mouvement permet d'apprécier quels sont les fondements d'un tel attachement et comment se déploie l'engagement patrimonial ordinaire. 


\section{L'émotion comme moteur d'une action patrimoniale}

4 Le mouvement qui s'est enclenché après la destruction du parc par la tempête a rendu visible un processus plus souvent immergé : celui qui nourrit l'affection à un lieu. Ce lien est devenu perceptible à travers les réactions suscitées par la disparition des arbres en décembre 1999 et pendant plus de deux ans après ${ }^{4}$.

Dès le petit matin du dimanche 26 décembre, alors que le vent vient juste de s'essouffler en Île-de-France, l'émotion se déploie à Versailles. Elle se manifeste en premier lieu au château lui-même, tout d'abord à travers la stupeur et la tristesse des jardiniers qui découvrent les dégâts causés par le vent devant leur porte. Les troncs d'arbres multicentenaires dont la présence semblait immuable jonchent les allées. Le président de l'établissement public ${ }^{5}$, qui réside également sur place, se joint à eux. Il faut gérer une véritable situation de crise : avertir le préfet de la dangerosité du parc, faire un inventaire des dégâts, organiser les travaux pour rendre le site accessible au plus vite et répondre aux questions pressantes des journalistes qui, déjà, affluent au château alors qu'en cette période des congés de fin d'année, le personnel du château est plus rare. L'émotion est également perceptible en dehors du domaine : les riverains "pleuraient agglutinés sur les grilles» du parc aussitôt fermé, se souvient-on à Versailles. Mais parmi les témoignages recueillis au château comme en ville, certains relativisent l'événement. Les familiers du parc ont gardé en mémoire un précédent: durant l'hiver 1990, des trombes de vent répétées avaient causé des dégâts autrement plus spectaculaires puisqu'ils avaient touché les abords du château. Replantés depuis avec de jeunes arbres, ils ont mieux résisté cette fois-ci.

6 Les pleurs et les témoignages de compassion rencontrent néanmoins la préférence des médias qui retracent l'événement. L'affliction et la consternation in situ se trouvent ainsi propulsées hors du château, accompagnées de toute une rhétorique de la catastrophe: "C'est une vision apocalyptique $»^{6}$, explique l'hebdomadaire local Les nouvelles de Versailles après une interview avec l'un des deux jardiniers en chef du château. Une semaine après, un jardinier raconte son émotion aux lecteurs du Pèlerin Magazine: "Le plus désolant, c'est le sentiment d'impuissance. Derrière ma vitre, je voyais ces énormes arbres, plus que centenaires, s'abattre comme des allumettes. Et c'était chaque fois un déchirement ${ }^{7}$. Cette émotion est d'autant plus palpable que les témoignages sont accompagnés d'images aux contrastes saisissants: des branchages enchevêtrés bouleversent le tracé rectiligne des allées du château, des troncs jonchent le sol au pied d'une statue ou d'un bassin, eux, miraculeusement intacts (ill. 2). 


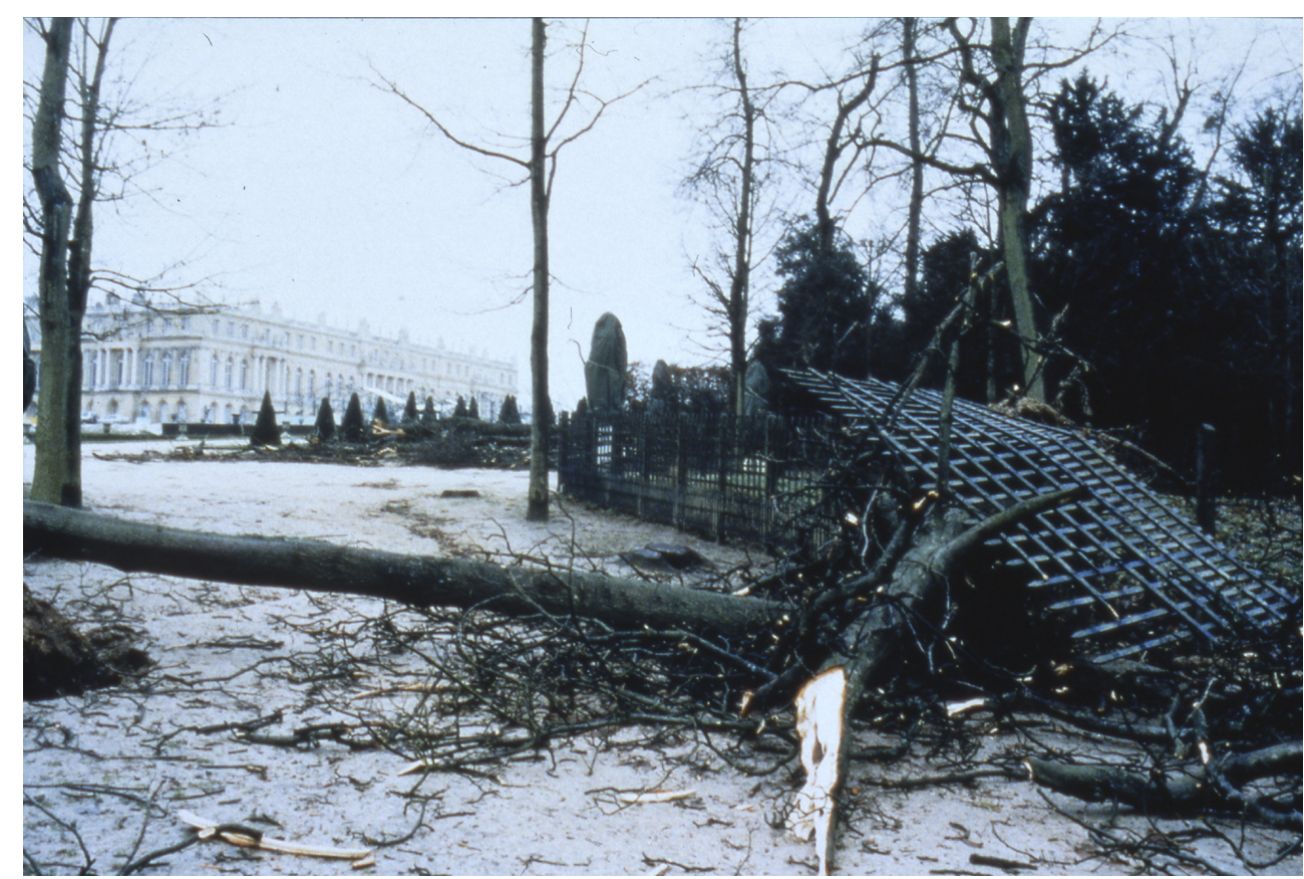

Photographie du parc sur le site Internet du château de Versailles, http://

www.chateauversailles.fr:tempete/Fr/souscription.asp lors de l'appel à souscription internationale, 1999-2001

Cl. J.-M. Manaï.

7 La presse écrite, les reportages télévisés ou l'Internet réactivent et amplifient l'émotion avec le temps puisque le renouveau du printemps, les plantations d'automne, les anniversaires de la tempête jusqu'en 2002 sont autant de prétextes commémoratifs. La mécanique patrimoniale s'enclenche sur ce substrat émotionnel.

8 L'émotion qui motive l'attention accordée au patrimoine végétal vient en écho à celle provoquée par la découverte des dégâts de deux manières différentes. Quelques jours après la tempête, Jacqueline écrit ainsi au président : « Je me permets de vous adresser cette lettre pour vous témoigner, ainsi qu'à vos collaborateurs, mon émotion face à la tristesse que vous avez éprouvée. Bien que ma région, l'Aquitaine, ait été gravement touchée le lendemain par les mêmes éléments déchainés, il est impossible de rester indifférent au désolant spectacle qui s'offrait à nos yeux en découvrant l'anéantissement de ce bien inestimable.» Chaque reportage diffusé ensuite sur Versailles est suivi d'un afflux de lettres au château: "L'idée m'est venue lors de l'émission "Des racines et des ailes" du 17 mai ", explique Danielle. La comparaison avec l'émotion éprouvée face à une perte similaire, subie dans son propre jardin ou dans un parc urbain près de chez soi, éveille la compassion. Ce qui a été vu ou entendu la veille « touche » des individus géographiquement dispersés et les rassemble dans un rituel de la solidarité compassionnelle qui se matérialise à travers des dons. Mais ce type d'émotion se dilue très rapidement dans le temps. Lui succède alors une émotion nourrie du vécu personnel et sans lien direct avec la tempête, si ce n'est une relative concomitance : Claude associe son don à l'arrivée d'Auriane et Mathilda, «petites filles nées l'année de la tempête». Christine dédie «cet arbre à [sa] nièce, Marion, à l'occasion de son baptême, prévu le 6 février prochain ». Les deux arbres financés par Bernard seront un cadeau pour de jeunes mariés. Anne et Pierre veulent honorer la 
disparition de leur grand-père: «En décembre dernier, la tempête emportait de nombreux arbres. Quelques mois auparavant, notre grand-père était, lui aussi, emporté par le temps. » La coïncidence physique ou mémorielle de deux événements aléatoires fait force de nécessité. Naissances, décès, mariages, communions, anniversaires sont autant de rituels de passage qui donnent lieu à des cérémoniels familiaux et qui trouvent, grâce au don patrimonial, une portée symbolique élargie ${ }^{8}$. Chacun peut s'y présenter selon ses attributs identitaires : en tant que parent, enfant, versaillais ou encore amoureux de la nature ou passionné d'histoire. Ce n'est toutefois pas au nom d'une caractéristique sociale donnée que l'on se mobilise mais parce que, grâce à l'émotion éprouvée, sont révélés ses propres attributs identitaires. L'action ne rassemble donc pas un "nous» préconstitué, elle transcende les catégories socioculturelles préétablies au profit d'une appartenance diffuse à l'humanité.

\section{Ordinaire ou tragique : l'émotion en partage}

9 Le don fait à l'établissement public du château et la lettre qui l'accompagne permettent d'établir des fondements identitaires. La tonalité émotionnelle perceptible dans les brèves lettres adressées au château traduit en effet le plus souvent des sentiments socialement éprouvés par tout un chacun : on partage sa joie d'accueillir un nouveauné dans sa famille, on honore la mémoire d'un aïeul disparu en donnant « des racines à sa mémoire » comme l'écrit Anne-Pierre. Les changements de positionnement générationnel et chronologique auxquels les arbres sont fréquemment associés renvoient au déroulement d'un fil biographique linéaire, inscrit dans la normalité : entrer dans la vie, grandir, se marier, avoir des enfants, des petits-enfants puis mourir. Chaque étape est marquée par la mise en forme d'un cérémoniel où le partage des émotions est de rigueur. En l'associant à la plantation d'un arbre, la joie ou la tristesse se diffusent et permettent à tout un chacun de partager la portée sociale de son geste grâce au bref commentaire envoyé avec le don. La contrepartie promise d'un parchemin calligraphié au château sera la preuve de son bon déroulement (ill. 3). 


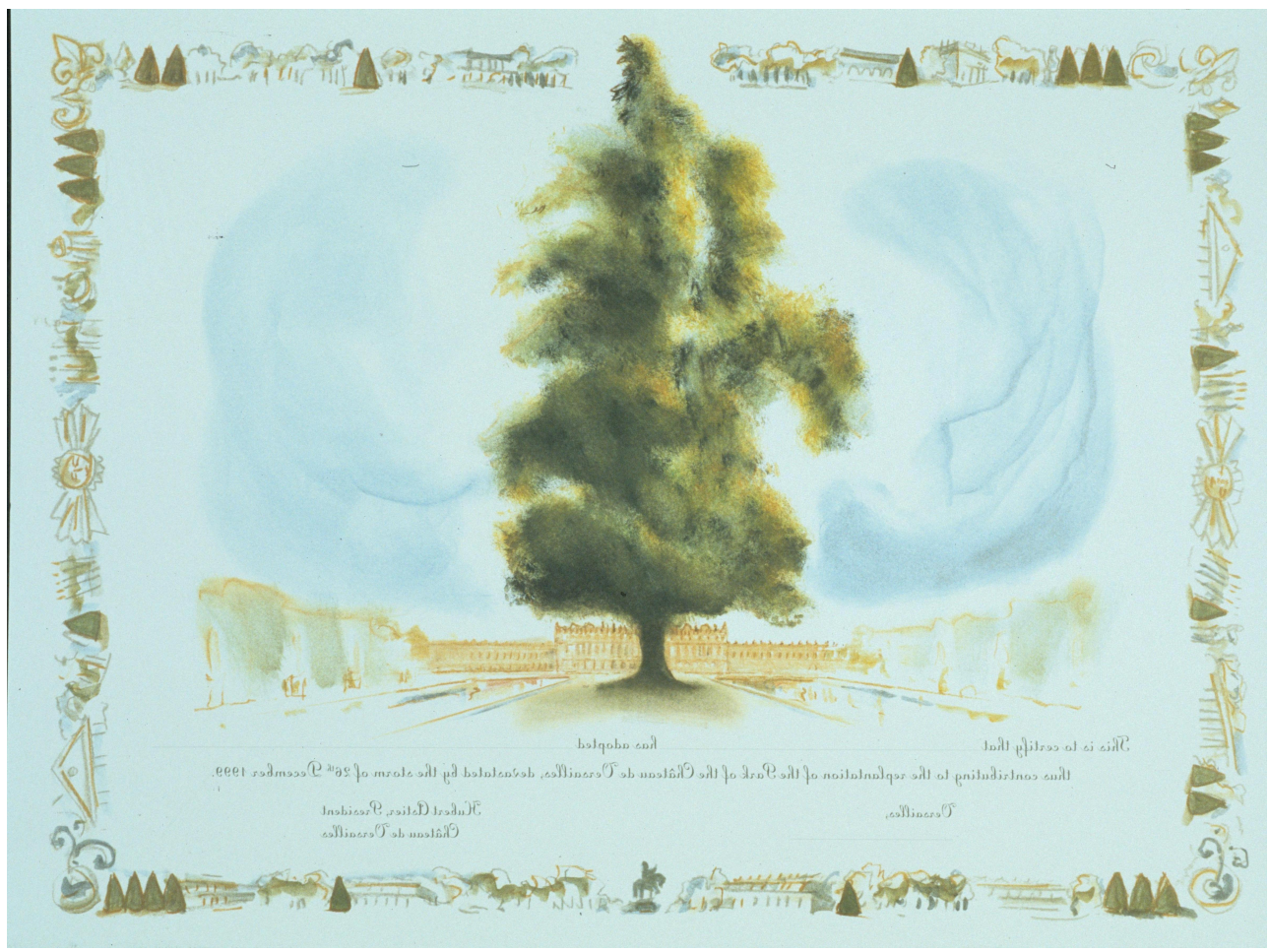

(c) Château de Versailles

Mais les lettres des donateurs amènent parfois ceux qui les lisent au château à partager une souffrance plus lancinante, personnelle et profonde. Des événements tragiques, comme la mort de Sandie, Frédéric et leur maman dans un accident "à cause d'un chauffard ivre ", sont aussi évoqués. Une mère, dont la fille de 23 ans est décédée d'un cancer l'année de la tempête, raconte « avoir souvent promené sa fille dans les allées du parc ». L'arbre adopté permettra «à d'autres jeunes d'en profiter à nouveau » expliquet-elle. Comme la tempête, le drame qui touche un enfant fait figure d'injustice. Ces événements paroxystiques remettent en cause un ordre que l'on pensait immuable : la pérennité des arbres centenaires profondément enracinés dans la terre et la succession des générations qui veut que les plus âgés meurent avant les plus jeunes. Dans le partage de ces émotions se rejoue une épreuve du destin qui ne peut laisser indifférent ${ }^{9}$. Les blessures profondes laissées dans la mémoire de ceux qui ont vécu ces tragédies marquent également ceux qui lisent leurs lettres au château, prêts alors à consentir quelques faveurs à ces donateurs. Alors que le choix des essences ou du lieu de plantation est habituellement imposé, les jardiniers seront en effet plus enclins à s'adapter aux demandes personnelles: un cèdre sera planté en mémoire du jeune Cédric, une plantation à la pièce d'eau des Suisses sera accordée pour une jeune défunte originaire du quartier Saint-Louis.

11 L'intimité se partage toutefois le plus souvent de manière moins violente avec le récit nostalgique de souvenirs associés au parc. La disparition des arbres convoque des épisodes biographiques. Ces souvenirs n'ont alors aucun lien avec la tempête ellemême : «Je suis âgée, invalide, [...], je n'ai aucun moyen de locomotion, c'est-à-dire que Versailles m'est à peu près inaccessible, hélas! Mais j'y ai passé des heures enchantées, il y a bien longtemps... », explique Rolande. Ce lieu, " m'accompagna dans mes rires et mes découvertes d'enfance, berça mes rêves et mon romantisme d'adolescent, j'y ai 
depuis promené ma nostalgie certains après-midi paisibles. La vision de ce désastre au lendemain de cette tempête a été pour moi une souffrance et m'a tiré des larmes", explique Jacques. C'est pour l'avenir que ces amoureux du parc proposent leur contribution. Le temps se déploie aussi bien à travers le souvenir d'expériences personnelles associées au parc que dans une projection vers le futur. La valeur de ce patrimoine devient perceptible à travers une expérience de reviviscence dont la traduction identitaire devient là encore explicite car, si l'on est devenu un familier du lieu, c'est qu'on l'a fréquenté avec assiduité, en tant que Versaillais ou à l'occasion de ses études, pour son travail ou parce qu'on a des proches dans ses alentours. Aussi la plupart des arbres adoptés le sont-ils au nom du donateur lui-même. L'identification du parc comme haut lieu du patrimoine se superpose ainsi à une identification de soi. L'émotion sert de liant entre le végétal et la personne au nom de laquelle l'arbre est planté, ce dernier permettant de repenser un parcours personnel et de lui donner une lisibilité concrète.

\section{Une catastrophe naturelle dans l'engagement patrimonial}

12 La mécanique affective de l'émotion patrimoniale n'est pas anodine dans ce processus d'identification de soi: outre une inscription du bien collectif dans le registre de l'intime, nous allons voir que ce processus implique une triangulation de la relation avec le patrimoine, ce qui favorise l'ancrage du «je» dans un «nous». Si personne n'évoque avoir pris sa décision après concertation avec ses proches ou son entourage, le don fait suite à la médiatisation de son utilité. Le mimétisme relationnel n'est pas de mise. L'engagement patrimonial implique toutefois la prise de parole d'un spécialiste. Dans sa lettre au président de l'établissement public, Monique explique : «Je vous ai entendu hier sur France Info et vous m'avez émue. Je suis passionnée par l'histoire de France et c'est pour cela que je suis heureuse de faire cette donation [...]. J'aimerais que l'arbre soit inscrit à mon nom. Je suis impatiente de pouvoir l'admirer.» L'action, personnelle, est motivée par la prestation médiatique d'individus lointains, que l'on ne fréquente pas personnellement. À l'inverse, le prosélytisme de certains auprès de leur voisinage s'avère inopérant en faveur des dons. L'intervention d'un tiers hors de la sphère relationnelle a pour corollaire l'absence de prescription de la part de l'entourage familier.

Des traits identitaires ne sont pas pour autant absents du propos; révélés par l'événement, ils alimentent le registre biographique, souvent sous l'angle d'une passion: on est un amoureux de la nature, jardinier, passionné d'histoire ou encore amateur de musique baroque. L'émotion patrimoniale se répercute ainsi toujours à deux niveaux, individuel et collectif. Sur le plan individuel tout d'abord, une émotion personnelle, intériorisée, est en quelque sorte retrouvée grâce à la cause patrimoniale. Percevoir chez autrui une émotion équivalente à celle que l'on se doit d'éprouver en pareille catastrophe offre un miroir de ses propres émotions. En souffrant avec, autrement dit en sympathie pour le parc, une émotion familiale peut acquérir une lisibilité sociale discrète puisqu'elle s'enracine sur un territoire d'État, sans aucun signe distinctif repérable dans le parc. L'arbre planté pourra être personnel comme devenir un bien familial symbolique, tout en restant propriété de l'État. En second lieu, la plantation, mise en acte d'un principe de responsabilité commune, favorise 
l'intégration dans un collectif élargi, devenu sensible grâce aux émotions partagées. La tempête est en effet apparue aux yeux de tous comme un signal d'alarme, « un signe du destin» explique Jean-Yves. La presse donne la parole aux préoccupations environnementales du chef indien Raoni : «Si vous continuez à détruire la forêt, la nature va se retourner contre vous $»^{10}$, prédit-il. L'Occident est responsable du désordre climatique dont la tempête est supposée être la conséquence. L'origine naturelle de la catastrophe dilue la responsabilité patrimoniale et favorise un consensus total en faveur d'une replantation auquel chacun a le devoir de s'associer. Ceci permet à des individus isolés de prendre part à une cause commune : «J'espère ainsi contribuer à la remise en état du domaine de Versailles [...] avec mes meilleurs encouragements pour la magnifique mission qui vous est confiée ", écrit un couple de donateurs. Des motivations "militantes et écologiques", comme celles de Christine sont rarement mises en avant, mais les donateurs ont quasiment tous recours aux termes de «reboisement» ou de "replantation» et non à l'idée de restauration, auquel la réparation d'un monument historique est d'habitude associée. L'action se trouve en quelque sorte d'autant plus légitimée qu'elle ne se fait pas en vertu de l'appartenance à un groupe social mais au profit d'un site prestigieux, dont la valeur parait universelle puisqu'on y associe l'avenir de la planète et des enfants qui en seront les dépositaires ${ }^{11}$ (ill. 4 et 5).

III. 4 : Plantation symbolique organisée à l'occasion d'une visite officielle d'une délégation du Québec, 07 nov 2000

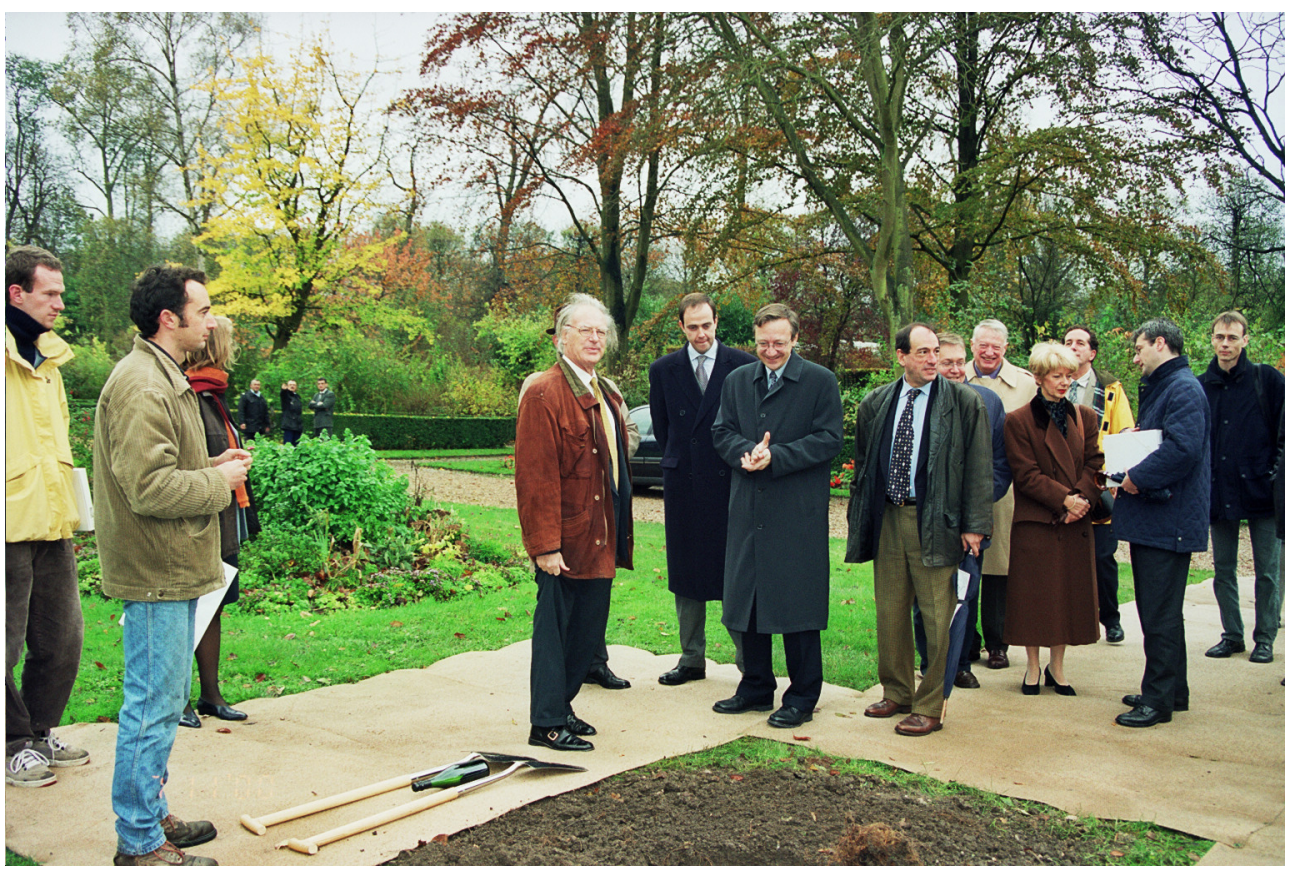

Cl. Joël Cottin. 
III. 5 : Replantation d'un bosquet organisée avec un lycée, 13 mars 2001

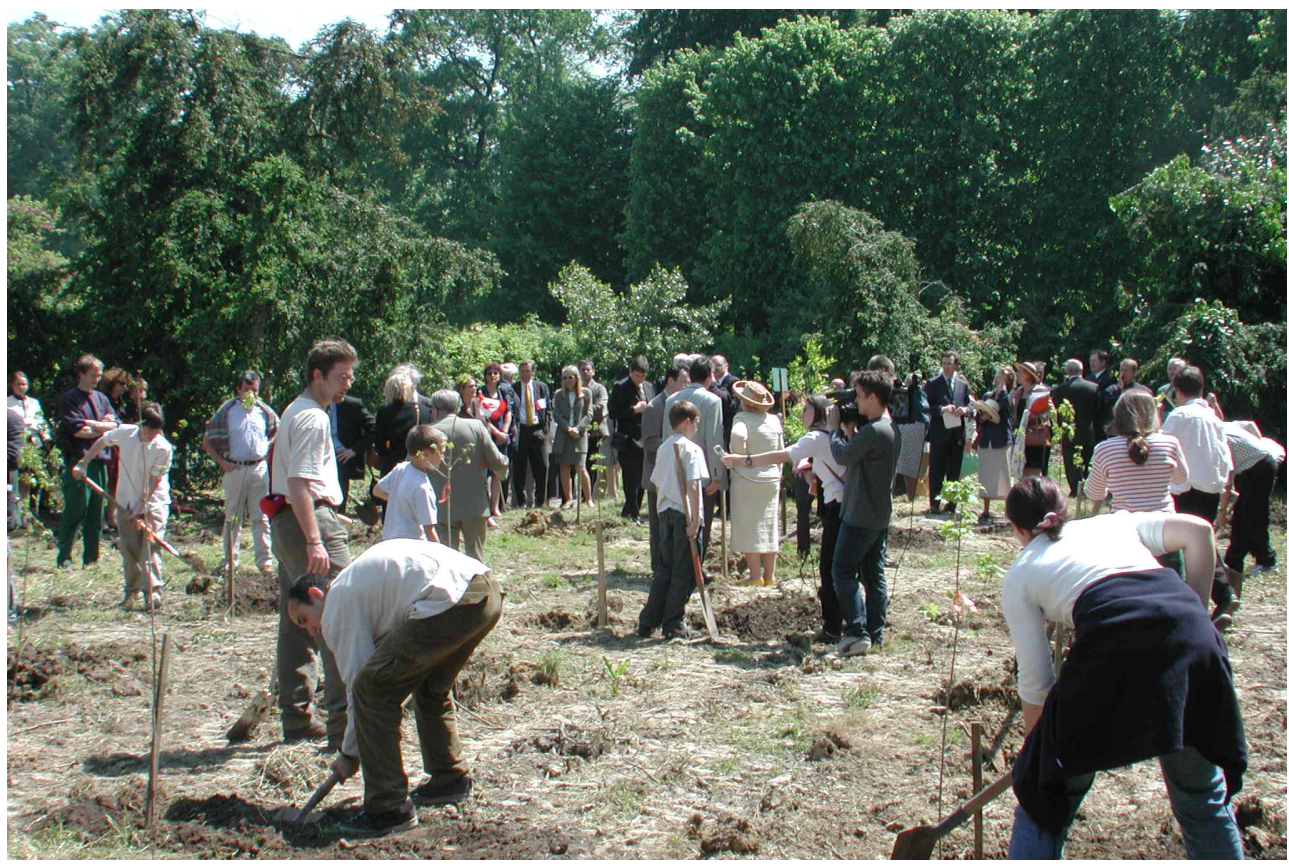

Cl. Joël Cottin.

14 C'est pourquoi il ne s'agit pas tant de restaurer un patrimoine que de " participer à la renaissance de la nature ", une "lourde tâche", un "travail exemplaire face à cette épreuve de la tempête », une "grande opération », un projet " considérable » comme l'écrivent les donateurs. L'accent mis sur le devoir commun apparaît ainsi en filigrane des discours et dans l'idée de "contribution " auquel chaque don est très souvent assimilé. Le geste de chacun intervient à la manière d'une goutte d'eau. L'insistance avec laquelle les donateurs présentent leur don comme «modeste» et leur regret de «ne pouvoir faire plus » les replacent dans un collectif universel, bien plus vaste que ne l'est celui de la sphère relationnelle du quotidien. Derrière la relation intime avec le parc, l'intervention médiatique permet une triangulation qui noue un autre lien, celui avec une humanité dont chacun partage des valeurs universelles.

15 Chacun peut ainsi s'approprier un archétype du patrimoine mondial et tirer profit de son prestige, sans avoir à l'expliciter. Le discours des experts habilités à prendre la parole dans les médias suffit à confirmer sa valeur: «Véritable architecture végétale prolongeant les lignes et les perspectives du château, les jardins de Versailles furent créés dans l'esprit de réunir l'art et la nature ${ }^{12}$. $"$ Les amoureux du parc qui se découvrent à travers le spectacle de sa destruction peuvent donner une portée biographique à leur geste sans avoir à l'inscrire dans une appartenance sociale ou culturelle a priori. En même temps, l'effet boule de neige de la souscription lancée par l'établissement public et dont la presse rend compte régulièrement permet à tous de prendre part à la mobilisation, au même titre que n'importe quel "citoyen du monde » ${ }^{13}$ pour enraciner sa propre expérience dans un collectif éphémère favorisé par la globalisation des appartenances ${ }^{14}$ : «Nous partageons avec vous ce témoignage de solidarité », explique Claude. Le consensus qui caractérise ce mouvement permet à chacun de réinventer son patrimoine et de l'ancrer dans sa propre vie. L'idée de catastrophe, les destructions occasionnées sur l'ensemble du territoire français sont un 
terrain propice à l'appropriation d'un objet du patrimoine, en dépit de l'éloignement aussi bien géographique que social.

\section{Des arbres victimes ou la personnification du patrimoine}

Les propriétés biologiques de l'objet interviennent également dans l'articulation du je et $\mathrm{du}$ nous mobilisée avec l'engagement patrimonial. Dans l'opération de communication, la formulation de l'appel à souscription lancé par le château, puis la manière dont les plantations vont être mises en scène renforcent les éléments propices à une identification au patrimoine végétal détruit.

17 Une véritable personnification des arbres tombés à terre en est le premier volet. Dans l'appel à souscription, il est en effet question du pin laricio "de Napoléon ", du cèdre « de Jussieu », ou encore du tulipier, « de Marie-Antoinette ». Ces arbres singuliers sont présentés comme des victimes, prestigieuses mais vulnérables au même titre que les personnalités auxquelles ils sont associés ${ }^{15}$. Ils feront d'ailleurs l'objet d'un véritable rituel mortuaire puisque, pour être montrés au public, ils seront lavés puis exposés devant le péristyle du Grand Trianon, les visiteurs étant tenus à une distance respectable par des barrières (ill. 6).

III. 6 : Arbres exposés devant le péristyle du Grand Trianon le jour de la vente aux enchères des arbres détruits par la tempête, 21 octobre 2000

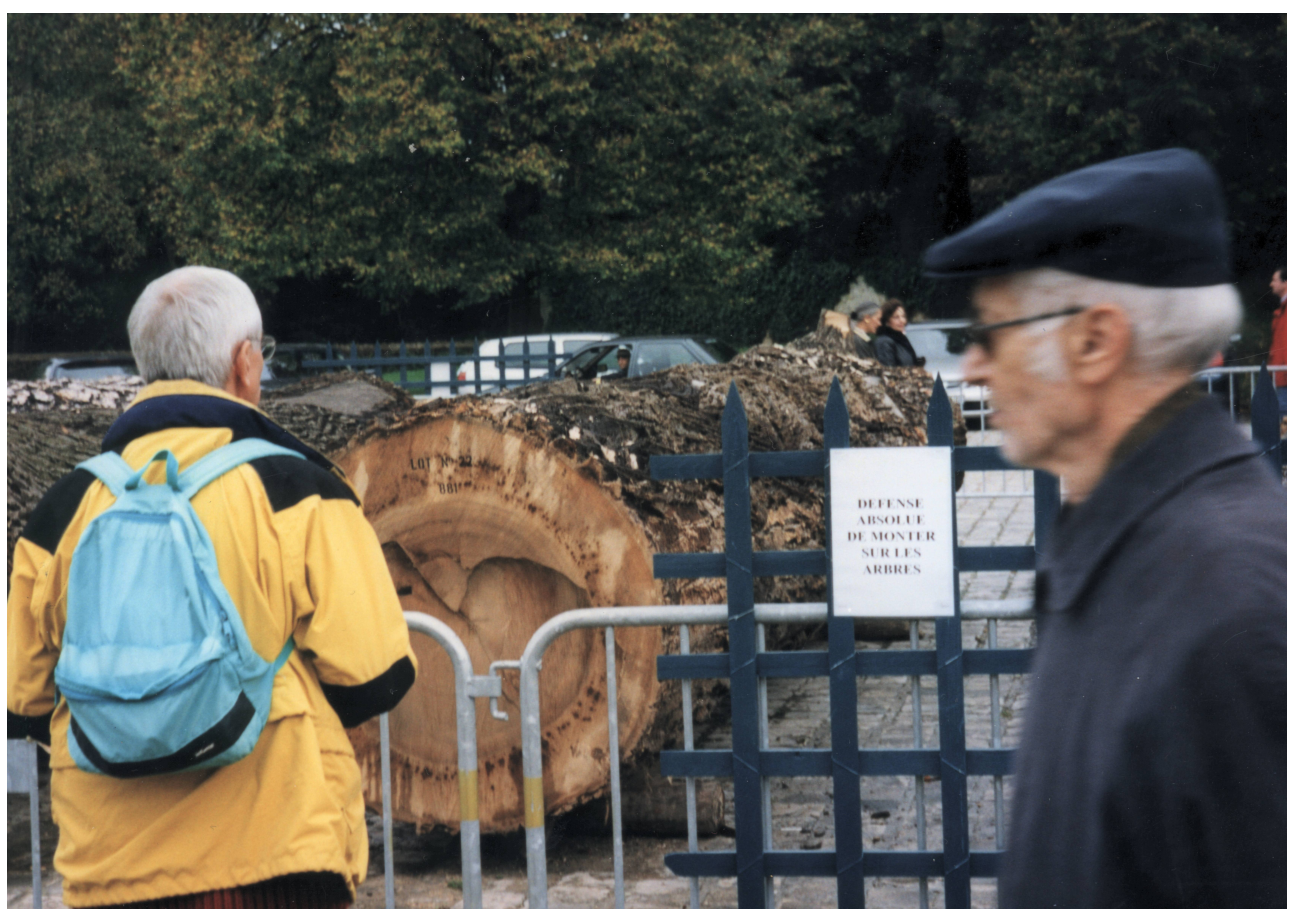

Cl. Martine Tabeaud

18 Ils apparaissent ainsi à la fois comme des reliques - celles des personnalités qui les ont côtoyés dans leur jeunesse - et comme des martyrs auxquels on vient rendre un dernier hommage. Leur corporalité se retrouve dans les propos des donateurs, qui veulent « effacer les immenses plaies» du parc. Thomas dit avoir été «beaucoup impressionné 
et très ému par tous ces arbres décapités, hachés ». L'arbre est un être qu'il faudra soigner - «Enracinez-le bien », demande Élodie - et dont on contrôlera la croissance " Nous aimerions connaître son emplacement afin de pouvoir le voir grandir ", précise Sylvie.

La souscription favorise également l'inscription des végétaux dans le registre de la parenté. En tant que supports des représentations généalogiques, les arbres s'y prêtent d'emblée particulièrement ${ }^{16}$. Mais en proposant à chacun d'en "adopter ", l'appel à souscription officialise cette prédisposition généalogique avec la promesse d'un parchemin calligraphié pour en attester. Aussi les donateurs ne manquent-ils pas de mentionner avec précision les noms de ceux pour lesquels ils adoptent un arbre. Paul et Denise le font au nom de leurs petits enfants, pour qu'ils puissent en se promenant dans le parc " avoir un souvenir tangible de la catastrophe et un peu de papy et mamy " plus tard. Catherine, soutenue par ses enfants, veut offrir quatre arbres en cadeau d'anniversaire à son mari: "Ces arbres représentent aussi nos quatre enfants", explique-t-elle. En tant qu'êtres vivants, ils sont des organismes biologiques qui vont évoluer, au même titre que leurs dépositaires. Leur superposition à la condition humaine autorise une appropriation qui délimite, au sein d'un territoire collectif, un espace personnel. Dans un "processus d'incorporation-intériorisation ${ }^{17}$, l'implantation rend alors visible le développement personnel sur une terre supposée originelle puisqu'on y trouve les fondements de sa propre culture.

Voué à devenir centenaire, l'arbre bénéficie d'une longévité qui couvre en moyenne trois générations. Cela en fait un témoin privilégié du passé tout en étant le garant de l'avenir. Sa croissance peut ainsi être mise en parallèle avec celle d'un être humain : «J'ai 87 ans... un jour, ayant pris mon envol, j'aurai le bonheur de venir à temps perdu, me perdre dans les frondaisons de mon arbre. J'ai tellement aimé cet admirable parc! Courage, cent ans, cela passe vite », explique Jean-Louis. L'enracinement dans un haut lieu du patrimoine, bien inaliénable et dont chacun a l'assurance qu'il restera accessible aux générations à venir, permet d'accéder à une temporalité immuable quels que soient les aléas familiaux. L'adoption d'« un arbre à leur nom permettra de pérenniser notre mémoire au-delà de nous-même » comme l'explique un donateur. Les émotions, qui ont pris corps dans des lieux et des temporalités différentes, se retrouveront dans un lieu singulier et voué à l'éternité. L'enracinement du monument végétal dans un lieu matérialise un collectif idéal, celui de la famille qui en est désormais le dépositaire : "Ce sont nos racines que le vent a soufflées et qu'il va falloir recréer », explique Sylvie. C'est pourquoi le regard vers le passé, auquel souscrivent plus volontiers les amateurs d'histoire, est paradoxalement rarement évoqué dans les correspondances. Les donateurs lui préfèrent le futur en évoquant leurs descendants. L'adoption est une projection dans l'avenir: Georgette veut « contribuer à rendre dans l'avenir toute sa beauté à ce merveilleux parc ». On plante pour soi mais aussi et surtout pour laisser une trace : «Il faudra que ma fille puisse au fil de sa vie contrôler la croissance », explique Arnaud (ill. 7). 


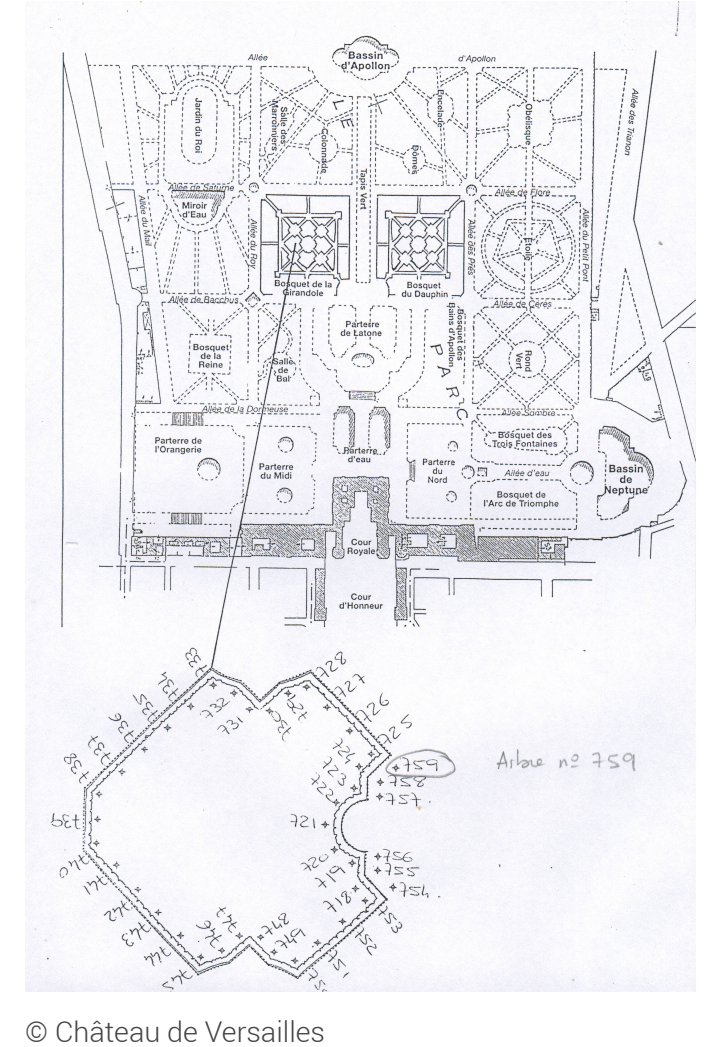

21 Connaître la localisation de l'arbre adopté afin de pouvoir « lui rendre visite » est par conséquent une condition essentielle, faute de quoi on préfère renoncer au don. Les donateurs s'inquiètent quand le plan de localisation de leur arbre tarde à leur parvenir. Plusieurs années après, ils restent d'ailleurs attentifs au développement de leur «filleul » végétal, terme d'ailleurs privilégié pour traduire cette parenté symbolique, et certains s'inquiètent au moindre signe de faiblesse de leur protégé, n'hésitant pas à alerter les jardiniers ou le service communication en cas de problème.

Le patrimoine apparaît propice au dévoilement d'une identité. Les émotions permettent à chacun de ressentir les attributs sociaux et généalogiques qui permettent de se définir en tant qu'individu singulier. À l'inverse, l'imaginaire d'un collectif universel permet de replacer chacun dans une appartenance globale. En réinventant ce patrimoine historique à partir de considérations affectives, humanistes et environnementales, il devient possible d'oublier les catégorisations socio économiques usuelles au profit d'un consensus identitaire fugace. L'attachement aux arbres historiques se traduit alors dans la lisibilité d'une histoire personnelle qui, via l'émotion partagée, prend place dans l'engagement patrimonial. À travers le végétal, le regard porté sur le parc laisse en arrière-plan la valeur matérielle de ce haut lieu du patrimoine mondial au profit d'une sensibilité naturaliste consensuelle et supposée sans frontière. L'introduction d'un fragment du patrimoine mondial dans la sphère de la vie privée a pour corollaire une mise en scène publique de l'intimité. Chacun peut, par l'intermédiaire du patrimoine, faire une expérience qui concilie à la fois l'irruption à la conscience de ses propres fondements existentiels et la rencontre avec un collectif diffus, rassemblé de manière éphémère autour de valeurs communes : l'importance de léguer un avenir à ses descendants et de respecter la mémoire du passé. 


\section{NOTES}

1. . Je remercie pour son soutien dans cette enquête l'établissement public du château de Versailles et tout particulièrement Pierre-André Lablaude, architecte en chef, Ariane de Lestrange, responsable du service communication, Joël Cottin et Alain Baraton, jardiniers en chef.

2. . Le Parisien, le 9 janvier 2000.

3. Cette recherche sur le parc de Versailles, qui a bénéficié du soutien du ministère de la culture, s'intègre dans un programme du LAHIC sur les «émotions patrimoniales ».

4. . L'analyse repose sur des témoignages oraux recueillis auprès de Versaillais un an après la tempête, ainsi qu'un corpus de 850 lettres reçues au château entre janvier 2000 et décembre 2001, soit environ un tiers des lettres envoyées et sur les articles de presse écrite ou télévisée diffusés depuis décembre 1999.

5. . Hubert Astier.

6. . Le 29 décembre 1999.

7. . Le 7 janvier 2000.

8. . Ils prennent la forme d'un "cérémonial médiatique " tel qu'a pu le décrire Clara Gallini à propos de l'usage des photographies familiales Voir Clara Gallini, («Le rituel médiatique », Gérard Althabe, Daniel Fabre et Gérard Lenclud (dir.) Vers une ethnologie du présent, Paris, 1992, éd. MSH, p. 117-126.

9. . Cette émotion ne peut être factice. En ce sens, elle évoque le «jeu de la Passion» mis en œuvre dans les théâtralisations de la Semaine sainte dans les rituels catholiques méditerranéens analysés par Claudine Fabre-Vassas. Voir Claudine Fabre-Vassas, « Le jeu de la Passion », L'Homme , 111-112, XXIX (3-4), p. 131-160.

10. Clarisse Josselin, « La nouvelle mise en garde du chef Raoni », Le Parisien, 02 mai 2000.

11. En cela, le procédé se différencie totalement de la mécanique de résolution des conflits dans les mobilisations publiques analysées par Laurent Thévenot, dans lesquelles « la mise en valeur d'un ancrage temporel et spatial, ainsi que la qualité des liens personnels mobilisés » intervient au contraire dans la mise en œuvre des émotions «Émotions et évaluations dans les coordinations publiques ». Patricia Paperman et Ruwen Ogien, La Couleur des pensées. Sentiments, émotions, intentions, Paris, École des hautes études en sciences sociales, 1995, 280 p., p. 161.

12. . Extrait du premier communiqué de presse diffusé après la tempête.

13. . Expression utilisée dans l'appel à souscription lancé par le service communication du château.

14. . Au triomphe de l'individualisme se mêle «la prolifération des identités collectives ». Voir Jean-Loup Amselle, «La globalisation, "Grand partage" ou mauvais cadrage ? ", L'Homme, 156, 2000, p. 222.

15. . Les arbres entrent alors dans le registre des "objets-personnes", au même titre que les œuvres d'art dans l'analyse de Nathalie Heinich. Nathalie Heinich, «Les objets-personnes: fétiches, reliques et œuvres d'art ", Sociologie de l'art, 6, 1993, p. 25-56.

16. . Christiane Klapisch Zuber, L'Arbre des familles, Paris, éd. de La Martinière, 2003, 215 p.

17. . Bernard Lahire, L'Homme pluriel. Les ressorts de l'action, Paris, Nathan, 2001, 271 p., p. 203-210. 


\section{RÉSUMÉS}

L'affection à l'égard d'un site patrimonial reste le plus souvent difficile à saisir. La tempête de 1999 et la destruction des arbres du parc du château de Versailles ont mis à jour un attachement très fort à l'égard de ce patrimoine arboré. L'émotion qui s'est déployée en faveur de sa restauration permet d'analyser les conditions d'un tel engagement. Cet article analyse quels sont les fils constitutifs d'une relation patrimoniale. Les lettres envoyées par les donateurs qui ont répondu à la souscription lancée par l'établissement public mettent à jour la portée identitaire d'un tel geste et sa déclinaison à la fois intimiste et collective. Adopter un arbre permet dans un même mouvement d'introduire un fragment du patrimoine mondial chez soi mais aussi de retrouver les fondements sociaux de sa propre histoire en allant rendre visite à son arbre dans le parc.

The affection felt for a heritage site is often difficult to measure. In 1999 the tempest that destroyed many trees in the park of the chateau of Versailles revealed a strong sense of attachment for this arboreal heritage. The emotions that were subsequently mobilised in the restoration of the park allow us to analyse something of the conditions of an engagement in favour of the heritage, looking at the strands which constitute a heritage relationship. Letters sent to the public establishment responsible for the park in response to the public subscription which it launched reveal the scale of this appeal and its components in terms of identity, with both intimate and collective aspects. Adopting a tree allows, at one and the same time, for the appropriation of a fragment of a world heritage site but also the rediscovery of the social foundations of the donors' own history when they go and visit their 'own' trees in the park.

Das tiefe Interesse für eine Kulturerbestätte bleibt öfters schwer verständlich. So erregte die Zerstörung der Bäume im Park von Versailles durch den Sturm von 1999 eine echte Leidenschaft für dieses Waldkulturerbe. Es entstand sofort eine tiefe Gefühlsbewegung, die die passenden Bedingungen für diesen musterhaften Einsatzwillen zugunsten der Restaurierung schuf. In diesem Aufsatz geht es um die Natur der Fäden, die eine so enge Beziehung geknüpft haben. Die Briefe der Teilnehmer an der von der öffentlichen Anstalt Versailles organisierten Spendenaktion sind repräsentativ für den sich identifizierenden Charakter einer solchen Anteilnahme in ihren privaten sowie kollektiven Varianten. Die Adoption eines Baumes bietet einem Spender Anlass, sich einen Anteil des Weltkulturerbes zu eigen zu machen und gleichzeitig die sozialen Grundlagen seiner eigenen Geschichte bei jedem Besuch zu seinem Baum in dem Park wiederzufinden.

\section{AUTEUR}

\section{VÉRONIQUE DASSIÉ}

Véronique Dassié, née en 1967, est docteur de l'École des hautes études en sciences sociales. Après un diplôme en ethnologie sur les représentations de la mémoire individuelle, elle a réalisé sous la direction de Daniel Fabre une thèse soutenue en 2007 sur la conservation privée des objets souvenirs, intitulée Pièces à confession. Ethnologie des objets d'affection dans l'espace domestique contemporain. Actuellement chargée de cours au département de sociologie de l'université François Rabelais à Tours, chercheur associée au LAHIC (IIAC Paris, UMR 8177) et au laboratoire CITERES (Tours UMR 6173), elle porte ses recherches sur le rapport au patrimoine à travers trois axes principaux : la genèse du lien patrimonial chez les jeunes enfants, l'articulation mémoire- 
patrimoine à travers les conservations d'objets ordinaires, l'émergence de nouveaux patrimoines (patrimoine arboré, patrimoine de l'immigration). Elle est l'auteur de plusieurs articles sur l'émotion patrimoniale déclanchée par la destruction du parc du château de Versailles :

« Décembre 1999 ; tempête sur Versailles : chronique d'une émotion », Ethnologies comparées, 2, « Mémoire des lieux », http://alor.univ-montp3.fr/cerce/revue.htm, 2002 ; « L'Amérique française enracinée à Versailles ", Encyclopédie du patrimoine culturel de l'Amérique française, http://www.ameriquefrancaise.org/index.php, 2008 ; « Le prix de l'émotion : la restauration du parc de Versailles après la tempête ", Journées d'étude Forêts et tempêtes, IHMC, Paris, École normale supérieure, janvier 2006. Publ. IHMC-CNRS, Cahiers d'étude, n 19, 2009. Ces travaux sur les musées et les conservations domestiques ont également donné lieu à diverses publications sur le patrimoine ethnographique : « L'antichambre du patrimoine ou l'itinéraire du linge de famille jusqu'au musée », Ce linge qui nous retient, Argenton-sur-Creuse, musée de la chemiserie et de l'élégance masculine, p. 21-70, 2002 ; «Conserver ou réanimer la Provence : le Museon Arlaten entre ethnographie et identité ", Anne-Solène Rolland et Hanna Murauskaya (dir.), De nouveaux modèles de musées? Formes et enjeux des créations et rénovations de musées en Europe XIXe-XXI ${ }^{e}$ siècles, Paris, L'Harmattan, 2008, p. 195-212 ; «Les fils de l'intimité », Ethnologie française, XXXIX, 1, 2009, pp. 133-140 ; « On the Ethnographic Museum in Arles. Are Popular Indigenous Artefacts Exotic?”, Journal of Museum Ethnography, $\mathrm{n}^{\circ} 22$ (avec Dominique Séréna-Allier), à paraître. Adresse électronique : lionver@club-internet.fr 